\title{
The serum amino acid profile in COVID-19
}

\author{
Alptug Atila ${ }^{1}$ (1) $\cdot$ Handan Alay ${ }^{2} \cdot$ Mehmet Emrah Yaman ${ }^{1} \cdot$ Tugrul Cagri Akman $^{3} \cdot$ Elif Cadirci $^{4} \cdot$ Burak Bayrak $^{1}$. \\ Saffet Celik ${ }^{5}$. Nihal Efe Atila ${ }^{6}$. Aycan Mutlu Yaganoglu ${ }^{7}$. Yucel Kadioglu ${ }^{1} \cdot$ Zekai Halıcı $^{4}$ - Emine Parlak ${ }^{2}$. \\ Zafer Bayraktutan ${ }^{8}$
}

Received: 21 March 2021 / Accepted: 17 September 2021 / Published online: 4 October 2021

(c) The Author(s), under exclusive licence to Springer-Verlag GmbH Austria, part of Springer Nature 2021

\begin{abstract}
The pandemic of the coronavirus disease (COVID-19) caused by SARS-CoV-2 affects millions of people worldwide. There are still many unknown aspects to this infection which affects the whole world. In addition, the potential impacts caused by this infection are still unclear. Amino acid metabolism, in particular, contains significant clues in terms of the development and prevention of many diseases. Therefore, this study aimed to compare amino acid profile of COVID-19 and healthy subject. In this study, the amino acid profiles of patients with asymptomatic, mild, moderate, and severe/critical SARS-CoV-2 infection were scanned with LC-MS/MS. The amino acid profile encompassing 30 amino acids in 142 people including 30 control and 112 COVID-19 patients was examined. 20 amino acids showed significant differences when compared to the control group in COVID-19 patient groups with different levels of severity in the statistical analyses conducted. It was detected that the branched-chain amino acids (BCAAs) changed in correlation with one another, and L-2-aminobutyric acid and L-phenylalanine had biomarker potential for COVID-19. Moreover, it was concluded that L-2-aminobutyric acid could provide prognostic information about the course of the disease. We believe that a new viewpoint will develop regarding the diagnosis, treatment, and prognosis as a result of the evaluation of the serum amino acid profiles of COVID-19 patients. Determining L-phenylalanine and L-2-aminobutyric levels can be used in laboratories as a COVID-19-biomarker. Also, supplementing COVID patients with taurine and BCAAs can be beneficial for treatment protocols.
\end{abstract}

Keywords Amino acid $\cdot$ COVID-19 $\cdot$ BCAAs $\cdot$ 1-2-aminobutyric acid $\cdot$ L-phenylalanine $\cdot$ Taurine

\section{Introduction}

The coronavirus (COVID-19) pandemic caused by SARS$\mathrm{CoV}-2$ has affected more than 100 million people worldwide as of 9 February 2021 and caused the death of more than 2.3 million people (https://COVID19.who.int/table) (Organization 2021). The number of cases increases worldwide day by day, and it still does not have any specific treatment.

Coronaviruses are the RNA viruses in the Coronaviridae family of the order Nidovirales and commonly seen in human beings and other mammals (Huang et al. 2020; Richman et al. 2020). In December 2019 in the city of Wuhan in China, many clinical cases emerged with an illness similar to viral pneumonia, the agent of which was unknown. A new

Handling editor: D. R. Jones.

Alptug Atila

alptug@atauni.edu.tr

Extended author information available on the last page of the article coronavirus called SARS-CoV-2 was detected as a result of the deep sequencing analysis conducted from the samples of the lower respiratory tract of these patients (Huang et al. 2020).

SARS-CoV-2 primarily targets the respiratory system of humans. The COVID-19 disease that it causes generally causes clinically mild symptoms such as a mild upper respiratory disease or an asymptomatic infection (Zhou et al. 2020). However, in some patients, it causes severe and potentially deadly respiratory diseases, including acute respiratory distress syndrome (Chen et al. 2020). The COVID19 disease is classified as asymptomatic, mild, moderate, severe or critical according to the clinical symptoms, radiologic involvement, and biochemical test results (Shi and Wang 2020).

In SARS-CoV-2, the emergence of a specific adaptive immune response is necessary for the host to eliminate the virus during the incubation and in the non-severe stages of the disease and prevents it from progressing to acute stages. 
However, the deformation of the immune response may cause the progression of the disease to severe stages and may even result in death (Yufang et al. 2020).

Studies have shown that the plasma levels of the biomolecules such as pro-inflammatory cytokines and tumor necrosis factor (TNF) $\alpha$ including chemokines, interleukins $1 \beta$ (IL-1 $\beta$ ), IL-6, granulocyte colony stimulating factor, interferon gammainduced protein-10, monocyte chemoattractant protein-1, and macro phase inflammatory protein 1- $\alpha$ Rise significantly in the immune response to SARS-CoV-2 disease (Huang et al. 2020; Liu et al. 2017; Li et al. 2020). However, there is insufficient information in the literature on how the serum profile of the amino acids, being the precursor of these and similar biomolecules playing a significant role in the immune response, is affected in SARS-CoV-2 disease.

It has been shown in many diseases that there could be significant prognostic factors in the increase and decrease of amino acids (Du et al. 2018; Hakuno et al. 2015; Hiraiwa et al. 2020; Mierzchala-Pasierb et al. 2020; Picca et al. 2019; Sookoian and Pirola 2014; Wang et al. 2011; Yanagisawa et al. 2015). It has also been shown in some clinical studies that clinical healing could occur by replacing the decreasing amino acids in significant diseases (Holecek 2010; Kudsk 2006; Liu et al. 2020; Reeds and Jahoor 2001). The detection of both cytokine profiles and the amino acid profiles in the diseases might be beneficial not only in previous estimation of possible organ damage but also in terms of developing different treatment strategies (Wang et al. 2011; Bi and Henry 2017; Manig et al. 2017; Mayers et al. 2014; Mondanelli et al. 2019).

Previous studies also focused on the importance of serum aminoacids during infections and also COVID-19 (Philips and Khan 2021; Paez-Franco et al. 2021; Rees et al. 2021). Determining the aminoacid profile in such acute conditions is important in terms of both diagnosis and supplementation (Cengiz et al. 2020) of the patients. Therefore, new studies focusing on serum amino acid profile and determining the changes are required and top of interest.

In this study, the patients who were determined to have SARS-CoV-2 infections via real-time PCR were clinically separated into groups including asymptomatic, mild, moderate, and severe/critical nasopharyngeal wipe samples were taken. The aim was to determine whether the findings showed changes in the serum amino acid profile between the healthy volunteers and the defined patient groups and to define the findings showing changes in correlation to the severity of the disease in the serum amino acid profiles of the patient groups.

\section{Materials and methods}

\section{Study design and participants}

111 adult COVID-19 patients with positive RT-PCR for SARS-CoV-2 receiving in-patient treatment between 1 July 2020 and 1 August 2020 in the clinic of Ataturk University Medicine Faculty Training and Research Hospital and 30 healthy volunteers were included in this case-control study ( $\geq 18$ age) (Erzurum/Turkey). The study was approved with Decision No. B.30.2.ATA.0.01.00/354 of the Ataturk University Medicine Faculty Clinical Research Ethical Board Commission.

Patients were separated into five groups according to the severity of the disease-asymptomatic, mild, moderate, severe, or critical-according to conducted studies and national guidance by a team of doctors consisting of the experts in infection diseases (Shi and Wang 2020; Bakanlı $\breve{g} 12020$; Wu and McGoogan 2020). The severe and critical groups were united in this study, and four groups were formed (Table 1). Asymptomatic patients could develop symptoms in a median of 4 days (3-7 days) after the first positive RT-PCR test (Sakurai et al. 2020). In this study, patients with no symptoms 4 days after the positivity of the first RT-PCR were accepted as asymptomatic.

The volunteers who came to the hospital for different complaints and whose RT-PCR results for SARS-CoV-2 were negative were included in the control group; these volunteers had similar demographic properties with the patients. 23 asymptomatic patients including 10 females and 13 males in the age group of 21-60 were included in Group 1. 29 mildly affected patients including 10 females and 19 males in the age group of 19-65 were included in Group 2. 31 moderately affected patients including 11 females and 20 males in the age group of 21-78 were included in Group 3.28 severely/critically affected patients including 11 females and 17 males in the age group of 19-85 were included in Group 4. 30 healthy volunteers including 14 females and 16 males in the age group of 18-60 were included in Group 5.

\section{Data collection}

The epidemiological, clinical, lab, and radiological data of the patients were saved including demographic information, symptoms, findings, lab findings, computed tomographic (CT) chest scans, presence of comorbid diseases, past exposure, smoking information, hospitalization duration, and applied treatments (antiviral treatment, mechanical ventilation, organ failure).

\section{Laboratory procedures}

SARS-CoV-2 was detected with the real-time RT-PCR method in respiratory specimens from the patients. 


\section{LC-MS/MS Analyses}

\section{Serum-free amino acid measurement}

Blood samples were taken from the ante-cubital vein of all participants after one night of fasting and placed into gel-containing biochemical tubes. The tubes were then centrifuged at $3500 \mathrm{rpm}$ for $10 \mathrm{~min}$, and serum samples were stored continuously at $-80{ }^{\circ} \mathrm{C}$. On the day of analysis, the samples were taken from cold storage and allowed to reach room temperature.

For both the experimental and control groups, $50 \mu \mathrm{l}$ of serum samples was then placed in Eppendorf tubes. Vortexing was performed for $10 \mathrm{~s}$ by adding $50 \mu \mathrm{l}$ of internal standard. To each tube, $700 \mu$ l of amino acid solvent solution (Mobile Phase A, Mobile Phase B, v:v,1:4) was added. After each sample was vortexed for $1 \mathrm{~min}$, it was centrifuged at $4{ }^{\circ} \mathrm{C}$ for $8 \mathrm{~min} .0 .45 \mu \mathrm{m}$ filters were used for the separation and filtering of the supernatant. The LC-MS/MS system (Agilent 6460 Triple Quadropol, USA) was used to measure the samples. The conditions for the chromatography and mass spectrometry of LC-MS/MS used for separating and defining the amino acids may be seen in Tables 2 and 3 .

\section{Statistical analysis}

SPSS Statistics (IBM v.20) was used in the statistical analyses, and RStudio (v. 1.3.1093) programs were used in the visualization of the data. Firstly, factor analysis was applied to the data cluster consisting of the amino acid analysis results conducted on serum samples. No transformation process was conducted due to the fact that the variables had mostly normal distribution. A one-way variance analysis (ANOVA) test was conducted to determine whether the amino acids showed differences among the patient groups as a variable. Patient groups consist of five different groups as categorical variables: control, asymptomatic, mild, moderate, and severe.

Receiving operating characteristic (ROC) analysis was conducted for the purpose of assessing the diagnoses of amino acids in COVID-19 patients. In the diagnosis group used in the ROC analyses, the control group diagnosis value was analyzed as negative samples, and the COVID19 patients with all symptoms were analyzed as positive samples. The relationship of variables with one another was examined in the correlation analysis conducted with the Spearman method. After that, primary component analysis (PCA) was conducted for the purpose of decreasing the dimension of the data set by protecting the variability of the data set as much as possible. Components becoming prominent in each dimension were determined in the analysis conducted on five dimensions. Binary logistic regression 


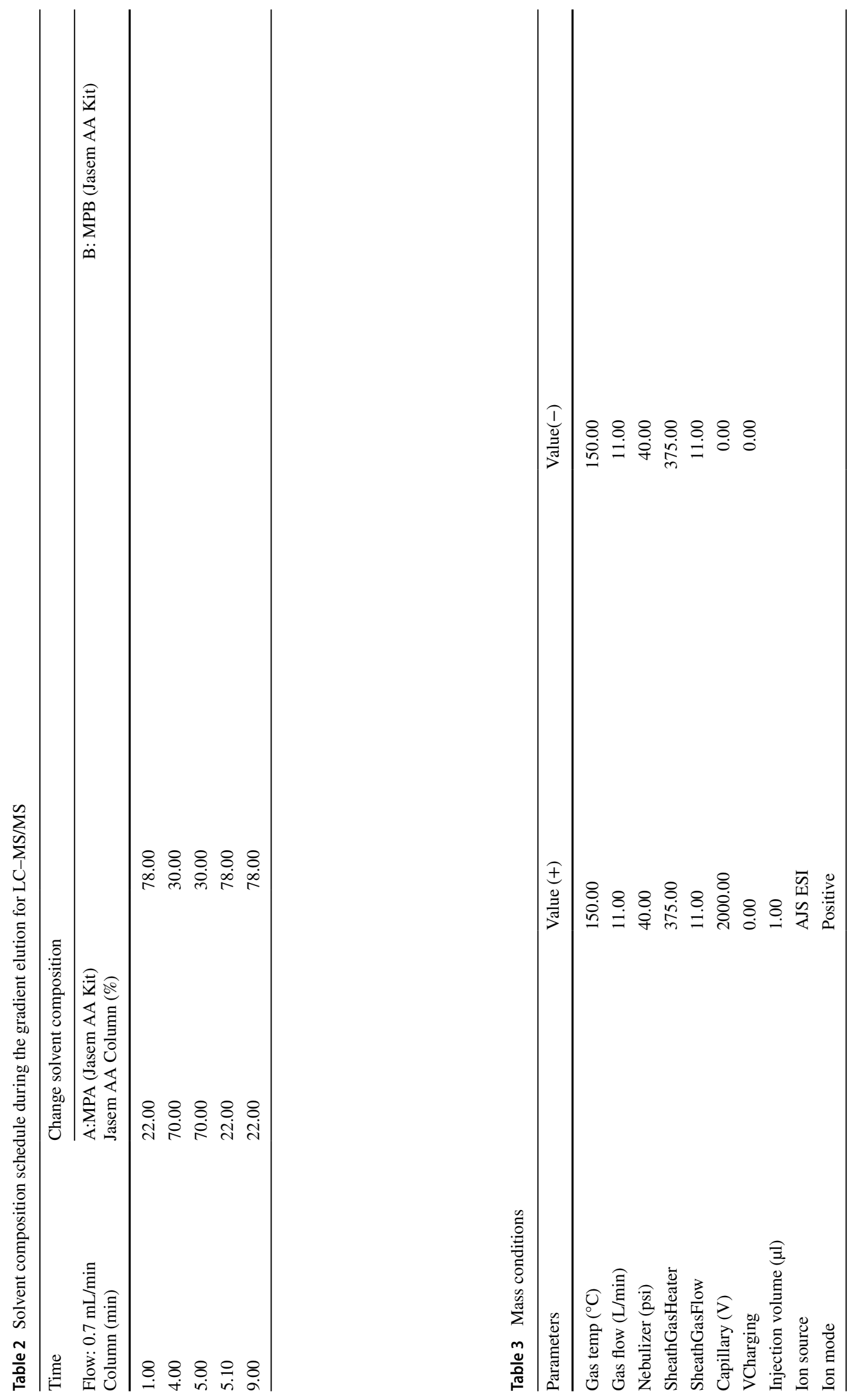


analysis was conducted to determine the separation power in the diagnosis group with these five components.

Boxplot graphs were prepared with a ggpubr package (Kassambara et al. 2020) in R software to visualize the amino acids with a significant difference among the patient groups. The GGally package (Mayer 2011) was used in drawing the correlation graph.

\section{Results}

\section{LC-MS/MS analyses results}

An LC-MS/MS analysis method with high precision, certainty, and accuracy and that could give high qualitative and quantitative data was used for the purpose of evaluating the amino acid profile in COVID-19 patients.

Serum-free amino acid levels belonging to a total of 142 individuals separated into control, asymptomatic, mild, moderate, and severe/critical groups were measured. 30 free amino acids were quantitatively assigned with LC-MS/MS in all participants to show the impact of SARS-CoV-2 infection on the serum amino acid profile. The linear regression equations and correlation coefficients for serum-free amino acid values are given in Table 4 .

\section{Anova (one-way variance analysis)}

According to one-way variance analysis, amino acids showing significant differences between the control group and patient groups and within the patient groups themselves were detected. It was determined that there were significant changes $(p<0.05)$ in the serum profile of 20 amino acids among the groups (Table 5).

Serum taurine and L-2-aminobutyric acid levels had significantly changed in all patient groups compared to the control group. The serum L-2-aminobutyric acid level increased in four separate patient groups compared to the control group. The serum taurine level significantly decreased in four patient groups. The trans-4-hydroxy L-proline serum level has showed a significant decrease in the mild/moderate and severe/critical patient groups but not in the asymptomatic group.

Significant differences were detected in the ethanolamine and L-citrulline serum levels of asymptomatic patients compared to the control group. While the L-citrulline serum levels decreased in asymptomatic patients, a significant increase was observed in the ethanolamine levels. In addition, the ethanolamine serum levels of the asymptomatic patients showed a significant increase compared to the mild and severe/critical patients.
The 3-amino isobutyric acid, L-glutamic acid and L-ornithine serum levels significantly decreased compared to the control group in the patients in the mild group.

The serum L-phenylalanine and trans-4-hydroxy L-proline levels significantly changed compared to the control group in the patients in the moderate group. In this respect, while the L-phenylalanine serum level increased to a significant level in the moderate group, the trans-4-hydroxy L-proline decreased.

When the serum amino acid profiles of the moderate and mild patient groups were compared; significant changes occurred in the serum levels of L-phenylalanine, L-tyrosine, L-glutamic acid, ethanolamine, L-aspartic acid, and L-ornithine. In this respect, the serum levels of these six amino acids belonging to the moderate patients significantly increased compared to the mild group.

L-threonine, L-proline and L-glutamine serum levels significantly changed in the severe/critical group patients compared to the control, asymptomatic, mild, and moderate patient groups. The serum levels of the three amino acids belonging to severe/critical patient groups significantly decreased when compared to other groups. Moreover, the serum levels of many amino acids showed changes along with the increase in the severity of the disease.

L-tryptophan, L-phenylalanine, 3-amino isobutyric acid, L-glutamic acid, L-alanine, L-glycine, trans-4-hydroxy L-proline, L-citrulline, L-histidine and L-ornithine levels belonging to the severe/critical group significantly changed compared to the control group. While the L-phenylalanine serum level belonging to the severe/critical group significantly increased compared to the control group, the serum level of all other amino acids decreased.

The serum L-glycine, L-glutamic acid, L-methionine and L-isoleucine levels revealed significant differences between the severe/critical and moderate groups. The L-glycine, L-glutamic acid, L-methionine, and L-isoleucine serum levels belonging to the severe/critical group significantly decreased compared to the moderate group.

While the serum L-phenylalanine levels of severe/critical patients significantly increased compared to the mild and asymptomatic groups, the serum L-histidine levels decreased.

\section{ROC analysis}

Eleven amino acids had the values of Area Under the Curve above 0.5 in ROC analyses. Two of them, in particular, L-phenylalanine and L-2-aminobutyric acid, had a high diagnosis power under a non-parametric assumption (Table 6, Fig. 1). It could be said with these results that L-phenylalanine and L-2-aminobutyric acid have a diagnostic separation power that could differentiate COVID-19 patients from control samples. 


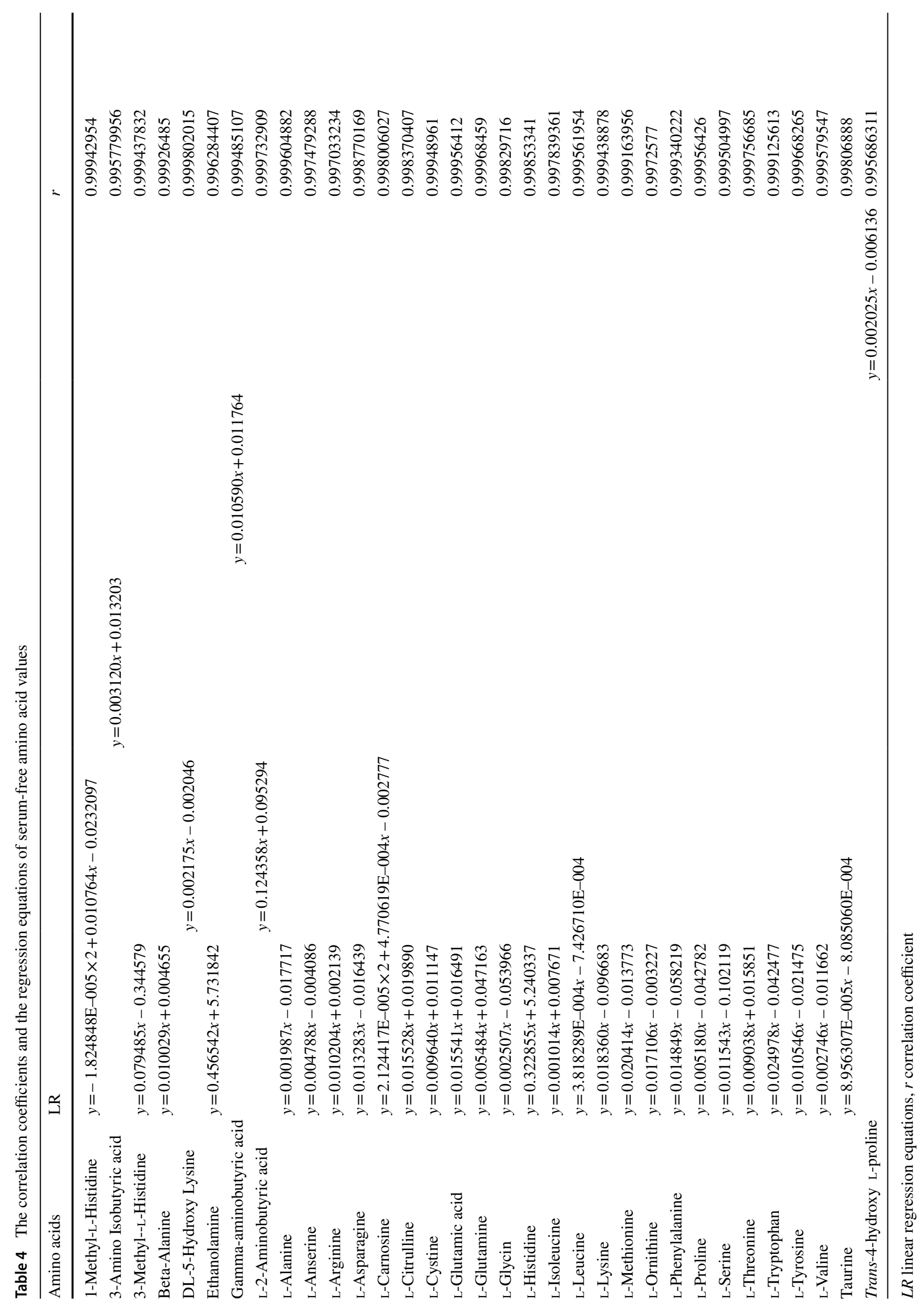




\section{PCA (Principal component analysis)}

PCA analyses were gathered in five principal components whose eigenvalue were 1 or higher for 33 amino acid variables. $61.5 \%$ of the five principal components with the highest variance ratio are explained in Table 7 . The results of the Kaiser-Meyer-Olkin (KMO) used in the measurement of sample sufficiency and the results of the Bartlett test used in the evaluation of global are shown in Table 8. The minimum KMO coefficient of the sample meets 0.5 (0.843), and it was seen that the used data set was appropriate for PCA analysis. The statistical significance of the results of the Bartlett test $(p<0.05)$ indicates the global of the attained results.

The five amino acids with the highest variability in five component vectors were determined by the PCA results and were used in binary logistic regression analyses. These five variables are L-valine, L-aspartic acid, 3-methyl-L-histidine, L-2-aminobutyric acid and L-serine (Fig. 2).

\section{Logistic regression}

The reference categorical data are the control data in the zero diagnosis group in logistic regression analysis $(n=30)$. The omnibus test of model coefficients was more significant than the $p<0.001$ ratio and showed that the developed model was a significant one. The Nagelkerke $\mathrm{R}$ square value of the developed algorithm was 0.507. While the developed regression model had correctly classified 17 out of 30 individuals in the control group, it could define 104 out of 114 patients. The total percentage of the estimation value is $85.2 \%$. The contributions of the variables to the diagnosis power are shown in Table 9. Here, L-2-aminobutyric acid is the variable with clearly the highest diagnosis power with a $p<0.001$ value and a 35.167 score value. When the significance value is taken into consideration, it could be concluded that the serum L-2-aminobutyric acid level had an ultimate impact on an individual becoming a COVID-19 patient. When its high positive score values were compared to the reference value, the increase in L-2-aminobutyric acid value was correlated with the course of the disease. This information could be used as the gold standard for the prognosis of the disease.

\section{Correlation matrix}

Correlation coefficient of Branched-chain amino acids were higher than $R>0.8$ for leucine, isoleucine, and valine among themselves. This finding shows that the three amino acids move together with a high correlation in serum samples, and three amino acids decrease in a correlated way in COVID-19 patients (see Fig. 4).

\section{Discussion}

This study examined the serum amino acid profile of COVID-19 patients with different severities, compared them to the profiles of healthy individuals, and revealed new targets for both diagnosis and treatment by revealing those that may have physio pathologic importance among these amino acids. Amino acids are necessary for the synthesis of many important molecules such as protein, hormones, and neurotransmitters, ensuring metabolic functioning (Wu 2009). Furthermore, amino acids ensure the immune response against diseases by being used in the activation of $\mathrm{T}$ and $\mathrm{B}$ lymphocytes, natural killer cells, and macrophages; in the cellular redox status, gene expression, and lymphocyte proliferation; and in the production of antibodies, cytokines, lymphokines, and cytotoxic substances. Many disease groups including oncologic diseases [hepatocellular CA (Watanabe et al. 1984), breast CA and lung CA (Cascino et al. 1995), colon CA (Leichtle et al. 2012), renal cell CA (Mustafa et al. 2011), cervical intraepithelial neoplasia and cervical squamous epithelium cell CA (Hasim et al. 2013)]; metabolic diseases [diabetes mellitus, metabolic syndrome (Tochikubo et al. 2016)]; and kidney diseases (Hong et al. 1998), sepsis (Vente et al. 1989), and nasal polyposis (Celik et al. 2019) and the relationship between serum/plasma amino acid profiles have been examined in biochemical terms in the literature. It has been asserted that the attained data could be used as a biomarker-treatment target in terms of the diagnosis of the disease, the course of the disease, and the efficiency of the treatment. Specifically, the significant ones among the amino acids analyzed in serum, and the potential candidates for diagnostic-therapeutic targets are discussed below.

Valine, leucine, and isoleucine amino acids have been studied in many groups as both branched-chain amino acid (BCAA) and essential amino acids. Basically, BCAA support has been observed to increase the anabolic pathways and could prevent cachexia in areas such as wound healing, ambustion healing, trauma, sepsis, and cancer. BCAAs transit to systemic circulation rapidly after protein intake. These characteristics are very important for muscles and the brain particularly, different from other amino acids. BCAA catabolism is conducted in very high ratios, especially in striated muscles, because branched-chain amino acid aminotransferase (BCAT) activity is very high in striated muscles. Branched-chain $\alpha$-ketoacid dehydrogenase (BCKD), the second enzyme in BCAA catabolism, is a multi-enzyme complex in the inner mitochondrial membrane and irreversibly decarboxylases the branched-chain $\alpha$-ketoacid (BCKA). 
The increase in BCKD activity is induced with TNF-alpha or endotoxins. Many studies have shown that the diseases increasing muscle breakdown such as sepsis, trauma, and surgical operations ensure that the glycine and alanine increases the BCKA activity produced by BCAA to mix with blood. In short, BCAAs have significant impacts on protein metabolism, glucose metabolism, and neurotransmission. There are neurohumoral changes such as systemic inflammatory response syndrome (SIRS) which includes cytokine production, sympathetic system production, and cortisol production). Protein breakdown increases in this hyper metabolic situation, body weight decreases, and, in a continuation of the process, death occurs with multisystem organ damage. In this situation, BCAAs become a very important energy resource for the muscles. Glycine level increases along with the increase in BCAA oxidation, and this are related to the immune system. In the preceding hyper metabolic situation, the glycine amount will decrease, and this will decrease the phagocytosis of the immune cells and the production of antibodies. The decrease in glycine levels shows that BCAA utilization increases significantly in muscles (Holeček 2018). BCAAs did not show significant differences in COVID-19 patients compared to the control group (Fig. 3). However, it was concluded that the correlation coefficients were greater than 0.8 (R, Spearman) for leucine, isoleucine, and valine according to the correlation analysis and they did decrease together from control to severe patient group (Fig. 4). This shows that the BCAA breakdown increases in muscles in an SARS-CoV-2 infection and also shows the necessity of clinical studies that would indicate whether BCAA support would decrease organ damage in patients with SARS-CoV-2 infection.

Of the amino acids examined in this study, taurine was particularly notable because, in the conducted ANOVA analysis, a decrease in taurine level in correlation with the disease was observed in all patient groups. Taurine is the free amino acid most abundantly existent in the body and with antioxidant, antimicrobial, and anti-inflammatory activity. Because taurine exists in leucocytes in high concentrations, its deficiency is thought to affect the immune cell functions. Taurine metabolism has been previously examined in the lymphocytes isolated from septic mice, and the contribution of the taurine pathway to sepsis physiopathology was shown (Dall'Igna et al. 2013). Because sepsis is a similar cytokine storm in COVID-19 physiopathology, the taurine level that is low in this study may be one of the causes or results of the distorted immune response in COVID-19 patients. Similarly, the amino acids found to be lower in COVID-19 patients when compared to the control group were trans 4-hydroxy-L-pyroline and L-pyroline. Proline is catabolized in many organs, including lymphoid organs via proline oxidase for the purpose of producing pyroline-5-carboxylate (P5C) and $\mathrm{H}_{2} \mathrm{O}_{2}$ (Wu
1997; Wu et al. 2005). This proline-P5C cycle regulates the proliferation of cells including the cellular redox situation and lymphocytes (Phang 1985). This could provide the cellular mechanism responsible for the role of proline in the protection of lymphocytes against apoptosis, stimulation of cell growth, and encouragement of antibody production (Duval et al. 1991). It has also been shown in previous studies that pyroline metabolism is distorted in patients with sepsis (Vliegen et al. 2020). Also, in the amino acid profile evaluation in the sepsis patients having severe cytokine storm and lung damage similar to COVID-19 and whose clinical findings are very similar to this study, the plasma levels of trans-4-hydroxy-L-proline and proline amino acids decreased (Su et al. 2017). The correlation with the decrease in trans-4-hydroxy-L-proline and pyroline levels and the distorted immune response in COVID patients is significant. Within this context, taurine, trans-4-hydroxy-L-proline, and pyroline appear to be the important amino acids in COVID-19 patients, both in terms of the follow-up of clinical status and diagnosis and being supportive in replacement treatments (Fig. 5). Namely, the change in amino acid profile shows correlation with the severity of clinical status. In critical COVID patients, SIRS can also occur as a result of catabolic state that is similar to SIRS.

In this study, other amino acids showing a significantly decreasing tendency in COVID-19 patients were L-threonine and L-glutamine (Fig. 6). Glutamine is the major energy substrate of immune system cells (Wu et al. 1991; Newsholme et al. 1999) and plays a vital role in the function and homeostasis of the immune cells. In addition, glutamine is necessary for the reproduction of the lymphocytes as an essential precursor for the synthesis of the purine and pyrimidine nucleotides (Ardawi and Newsholme 1983; Wu et al. 1992). The increase in the extracellular concentration of glutamine from $0-1$ to $0-5 \mathrm{mM}$ (a physiological level in plasma) increase lymphocyte proliferation depending on the dosage (Wu et al. 1992). The addition of $2 \mathrm{mM}$-glutamine to a culture environment prevents apoptosis, stimulates cell growth, and increases the production of antibody in lymphocytes (Franěk and Šrámková 1996). The existence of glutamine (0.5-2 mM) in physiological levels in the plasma modulates the production of cytokines by the monocytes and macrophages (Spittler et al. 1997; Yaqoob and Calder 1998). Similarly, the studies conducted in an in vitro environment and on the farm, animals support the idea that threonine has a role in the modulation of the immunity function (Su et al. 2017). It has also been found in previous studies that both threonine and glutamine have been found at decreased levels in the blood of the patients with inflammatory diseases such as sepsis. During sepsis in rats, there is increased synthesis of both mucins and gut epithelial proteins, and 


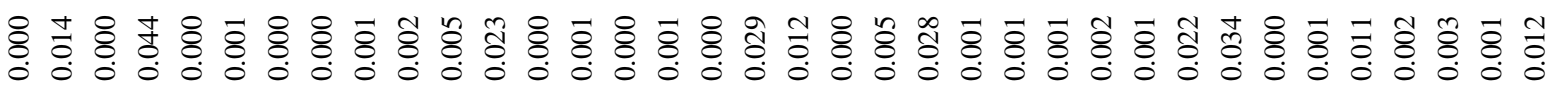

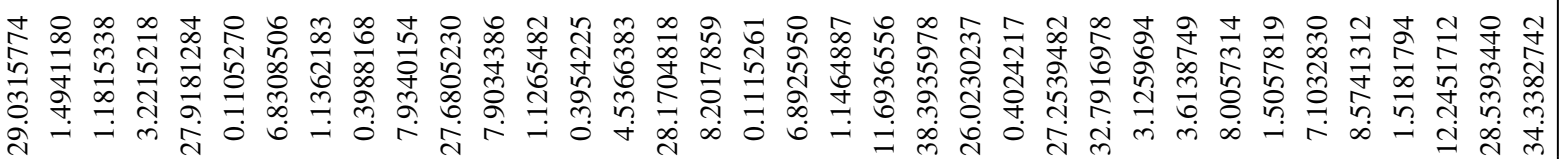

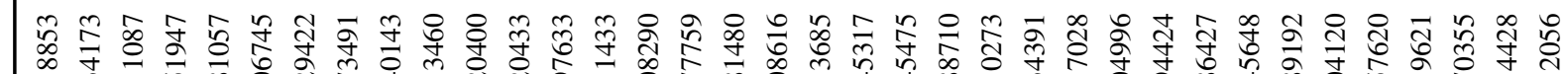

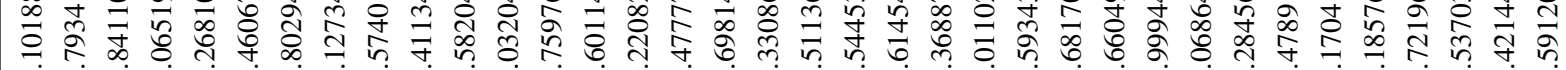

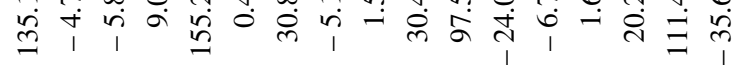

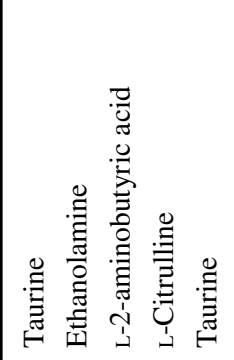

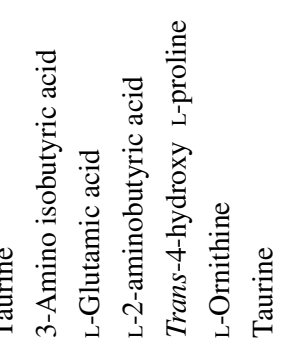

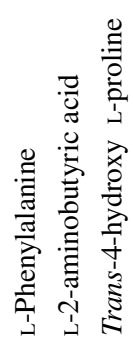

悬

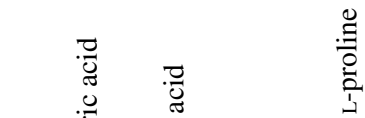

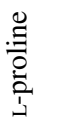

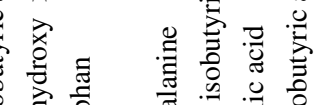<smiles>[CH]1[CH][CH]C[CH]1</smiles> 


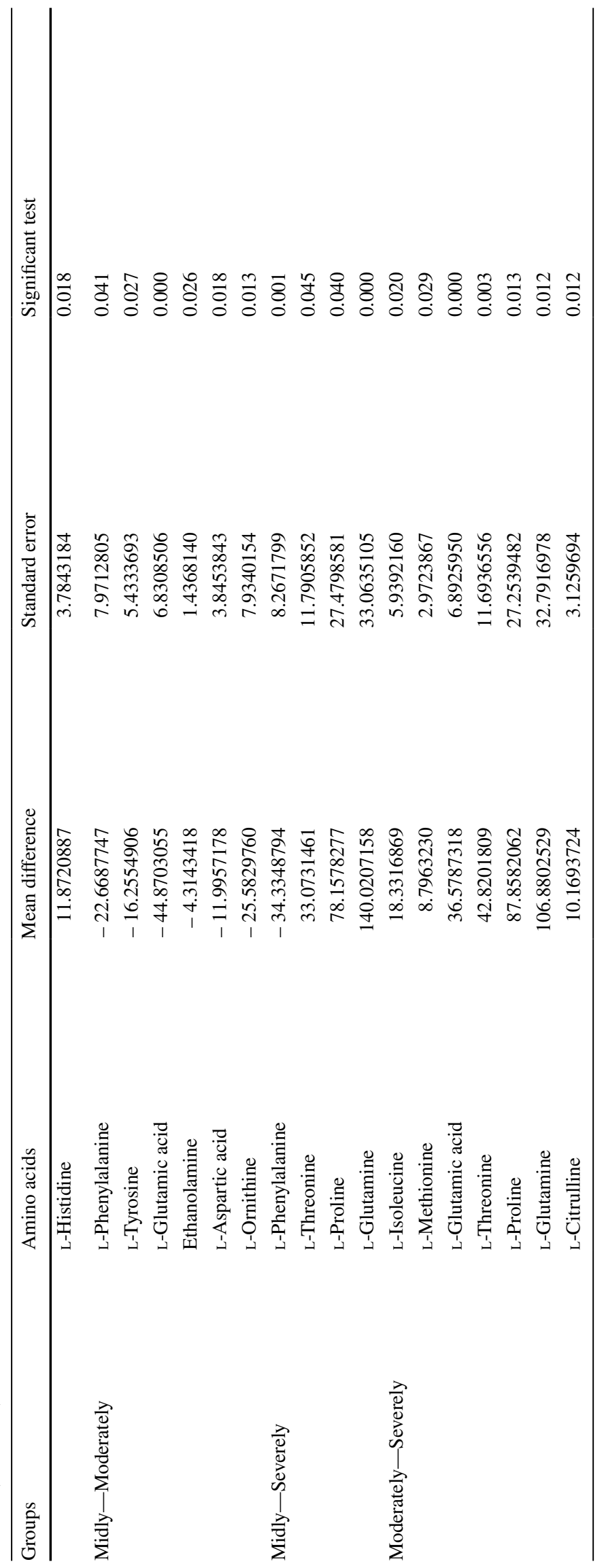

照 Springer 


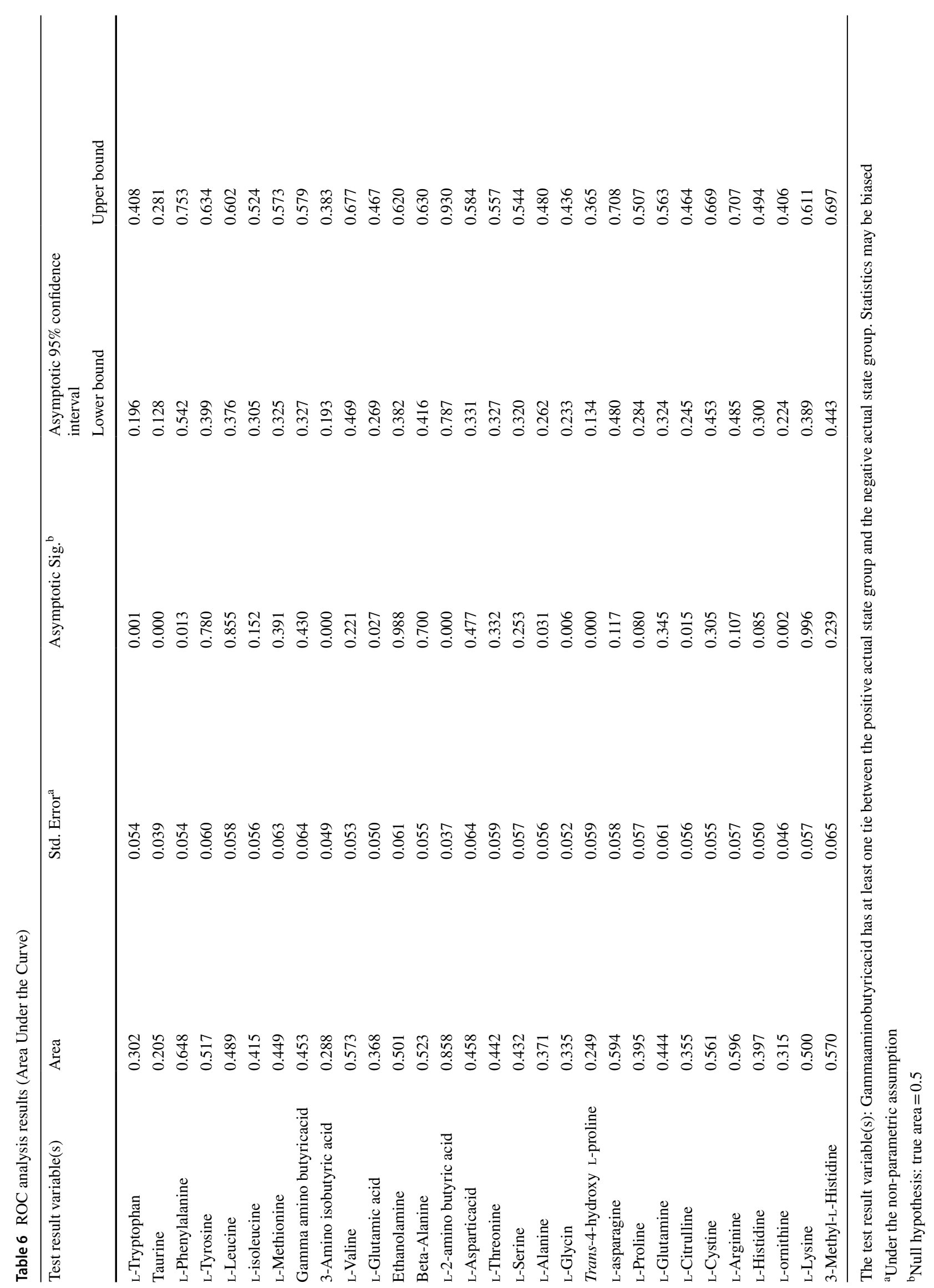




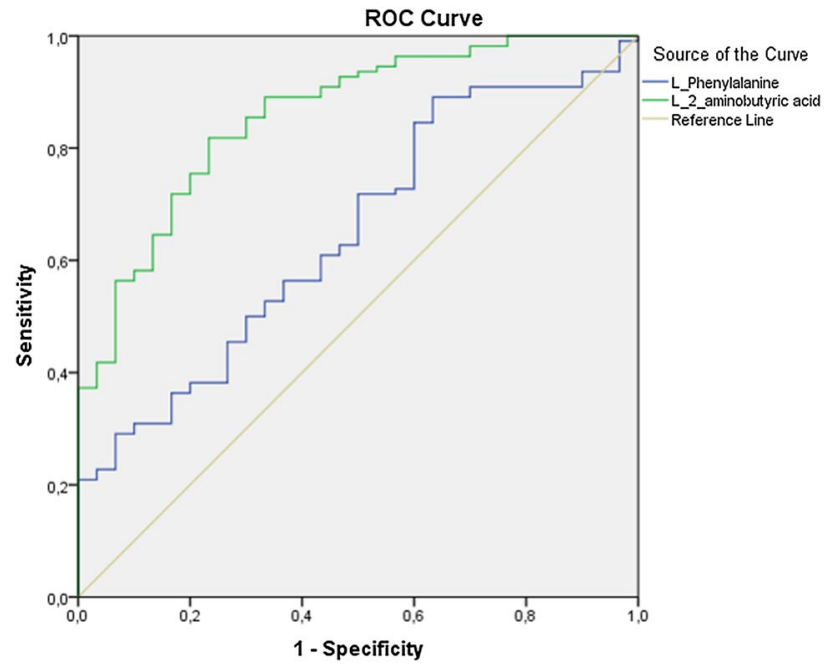

Fig. 1 ROC curves of L_phenylalanine and L 2-aminobutyric acid obtained by ROC analysis

this increases threonine demand to nearly threefold that of normal intake. Although extra requirements to support mucin and gut epithelial protein synthesis may also occur in the current challenge, the increase in amino acid activity in both liver and kidney indicated a probable increase in the rate of catabolism of threonine and glutamine. In this study, the protein catabolism increasing similar to the one in sepsis has increased the catabolism of threonine and glutamine, and this may have increased the need for amino acids.

In this study, L-histidine and L-citrulline were focused on as the last parameter in the general decrease observed in the amino acid levels of COVID-19 patients (Fig. 7). Histidine works as substrate for the histamine synthesis in macrophages, thrombocytes, dendritic cells, and $\mathrm{T}$ lymphocytes. The histamine produced from histidine regulates various physiological and immunological functions by activating various histamine receptors on the target cells. In addition, histamine mediates the thrombocyte aggregation and regulates the Th2 cell activity both by decreasing IL-12 production and by increasing IL-10 production. Histamine may also mediate the eruptions observed in COVID-19 patients as well as contributing to the inflammation occurring in COVID-19 (Demopoulos et al. 2020). Excessive histamine production in COVID19 patients may have caused the decrease in plasma histidine stores (Ho and Ray 2020). Similarly, citrulline is the precursor used in most cells in the synthesis of arginine and contributes to the formation of arginine nitric oxide (Wu and Morris 1998). The plasma concentrations of citrulline also clearly decrease in the situations of inflammation and sepsis (Bansal and Ochoa 2003). In a previous study that supports our findings, it was also shown that citrulline levels decrease in the plasma of COVID-19 patients, and this decrease is related to the inflammation severity of the system, particularly the gastrointestinal symptoms (Uzzan et al. 2020).

L-2-aminobutyric acid is seen as a gold standard in all the conducted statistical analyses. Also in previous studies, increased alpha-amino- $n$-butyric acid (ABA) was correlated with increasing total aminoacidemia, lactate, neutrophil concentration, creatinine, ammonia, osmolarity, glucose, and bilirubin and with a decreasing Fischer's ratio and peripheral $\mathrm{O}_{2}$ extraction (Chiarla et al. 2011). The author commented in this article, "Elevations in ABA seem to reflect liver damage; in as much as elevations in creatinine usually indicate kidney failure, it would seem appropriate to focus on comparison of ABA concentrations with those of enzymatic and other indicators of liver damage" (Effros 2011). In another study, the results showed that M. hyopneumoniae infection time-dependently altered the serum levels of selective amino acids and fatty acids. L-2-aminobutyric acid and long-chain fatty acids were markedly increased at 14 and $21 \mathrm{dpi}$ in inoculated pigs $(p<0.05)$ (Nair et al. 2019). According to the ROC analysis attained from this study, while the increasing L-2-aminobutyric acid level has shown that it could be valuable as a differential criterion in the diagnosis of COVID-19 disease, the increase in L-2-aminobutyric acid in correlation with the severity of the disease has shown in logistic regression analyses that it could be used regarding the prognosis of COVID19 disease.

To sum up the above information, it has been observed that there was an increase in the amino acid metabolism of COVID-19 patients, and a decrease was observed as a result of this catabolic activity, especially in leucine, isoleucine, valine, taurine, Trans 4-hydroxy L-pyroline, L-pyroline, L-threonine, L-glutamine, L-histidine and L-citrulline serum levels. However, although COVID19 caused a decrease in the general amino acid levels, interestingly, it caused a significant increase in L-phenylalanine levels when compared to healthy patients, and L-phenylalanine was notable as a differential marker for COVID-19 patients, according to ROC analysis. Alanine is an important substrate in the hepatic synthesis of glucose necessary for the ATP synthesis in leucocytes (Newsholme and Newsholme 1989), and therefore, it affects the immune response. There is significant evidence regarding the fact that $2 \mathrm{mM}$-alanine support to the culture environment in B-lymphocyte hybridoma-cells prevents apoptosis and increases cell growth and antibody production (Duval et al. 1991; Franěk and Šrámková 1996). Also, in a study conducted on septic sheep, the plasma L-phenylalanine levels significantly increased when compared to healthy sheep (Hoskin et al. 2016). 


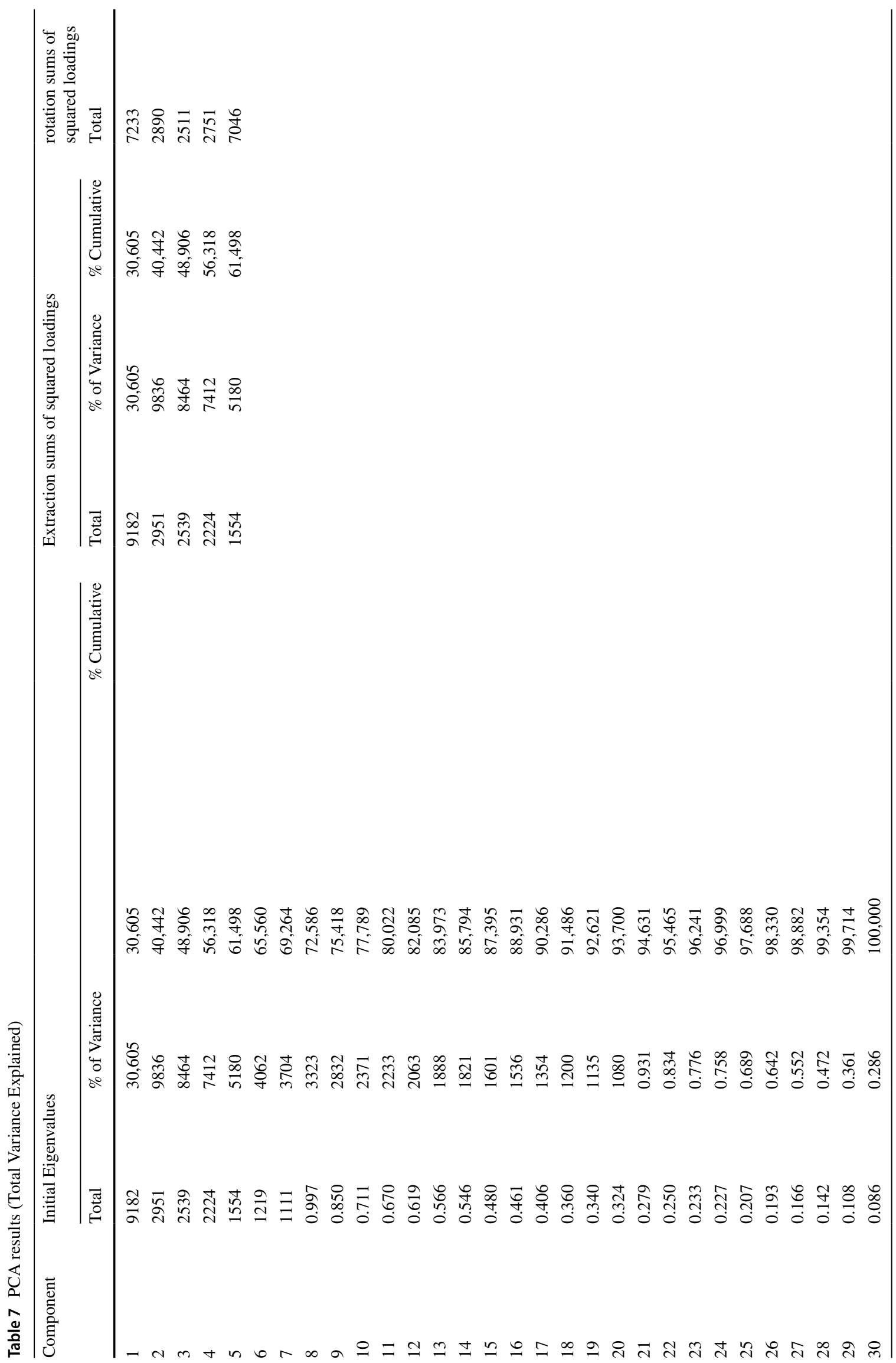


Table 8 The Kaiser-Meyer-Olkin and Bartlett test results of PCA analysis

\begin{tabular}{llll}
\hline Kaiser-Meyer-Olkin & \multicolumn{3}{l}{ Measure of sampling adequacy } \\
\cline { 2 - 4 } $\begin{array}{l}\text { Bartlett's test of } \\
\text { sphericity }\end{array}$ & Approx. Chi-Square & $d f$ & Sig \\
\hline 0.843 & 2522.220 & 435 & 0.000
\end{tabular}

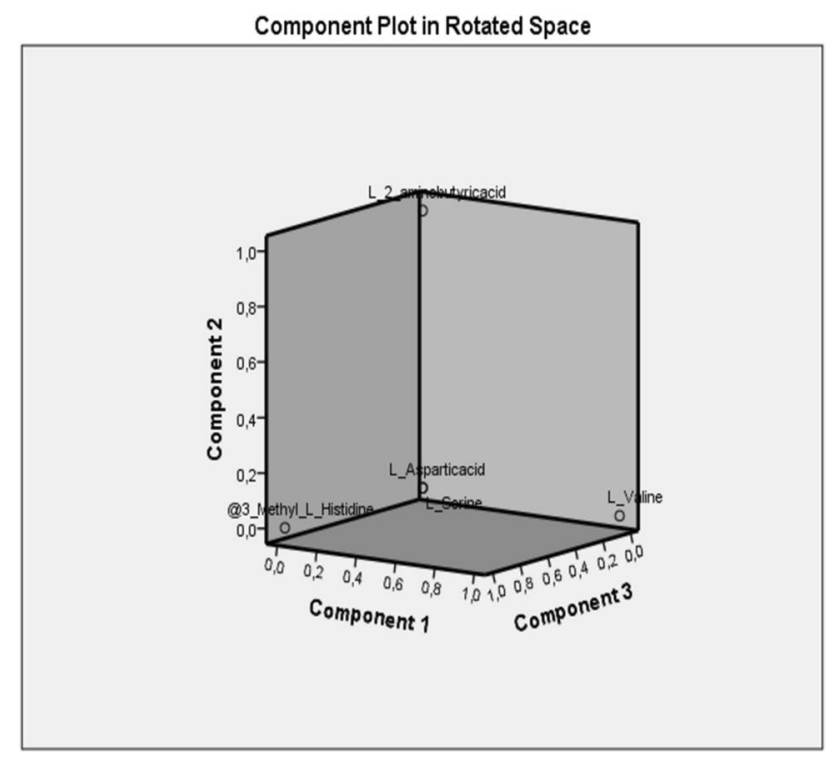

Fig. 2 Display of five prominent variables in PCA analyzes on rotated speace

The increase in the phenylalanine amount in HIV-infected patients was thought to be due to the distortion of gastrointestinal phenylalanine intirosine metabolism (Innocenti et al. 2020). In the study conducted on test subjects with sepsis, the plasma L-phenylalanine level was found to be high, and these values were reduced to normal in the treated groups (Cui et al. 2020). Huang et al. also specified that the increased phenylalanine levels show a correlation with renal failure and low albumin levels is an independent predictor of death (Huang et al. 2019). In this study, ROC analysis was increased and showed that L-phenylalanine could be valuable as a differential criterion in the diagnosis of the disease.

Consequently, COVID-19 affects the metabolic pathways of patients with a general destructive process and causes a significant decrease in the levels of many amino acids. The determination of these decreasing levels of amino acids may be a factor that would assist in diagnosis, and their replacement will also be valuable in terms of treatment protocols. The increasing parameters that could be used specifically as diagnosis criteria in COVID patients are serum L-phenylalanine and L-2-aminobutyric acid levels, and an increase 


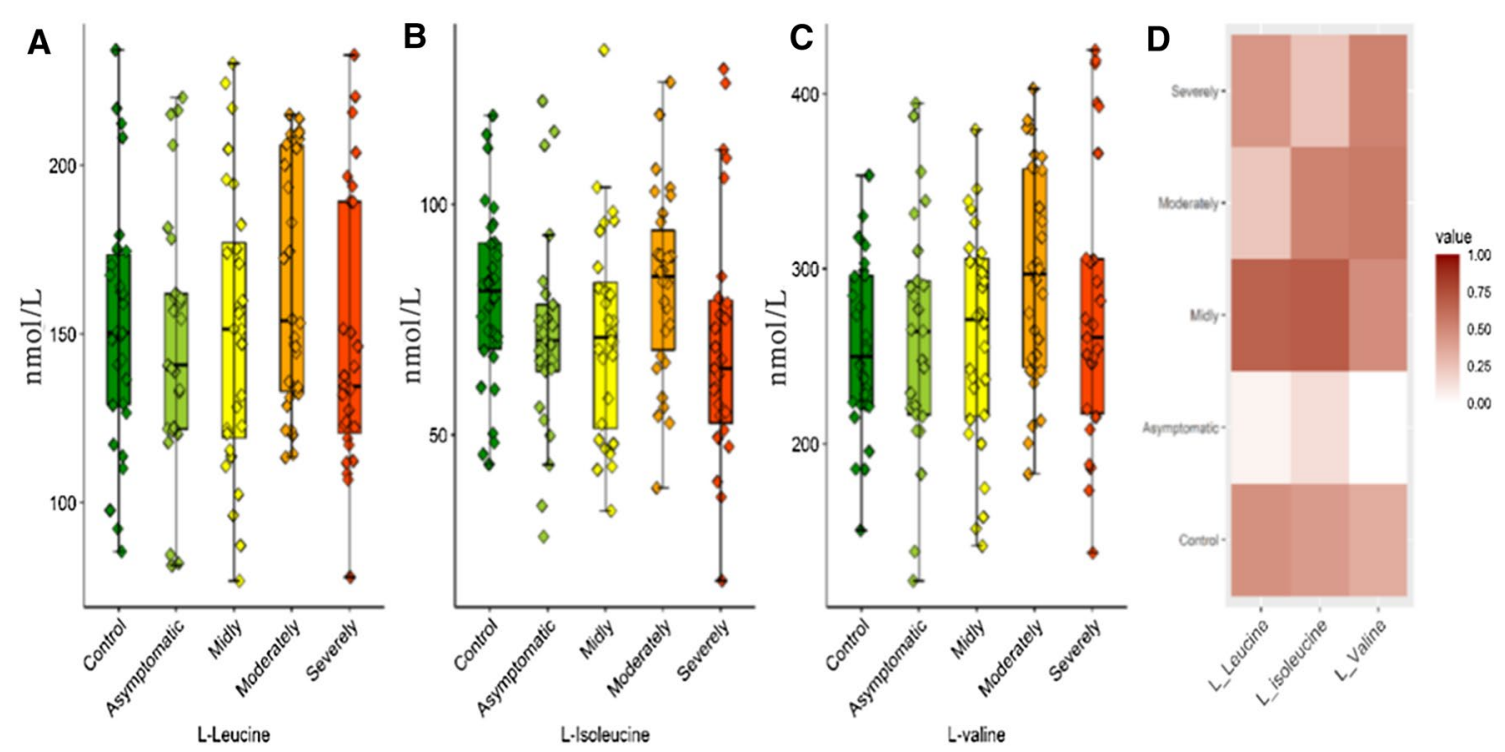

Fig. 3 Box plots graphs showing differences BCAAs levels a L-Leucine. b L-Isoleucine. c L-Valine. d Heat maps of BCAAs

Fig. 4 Spearman correlation matrix of serum aminoacids levels. Positive and negative correlations are indicated by blue and red colours, respectively

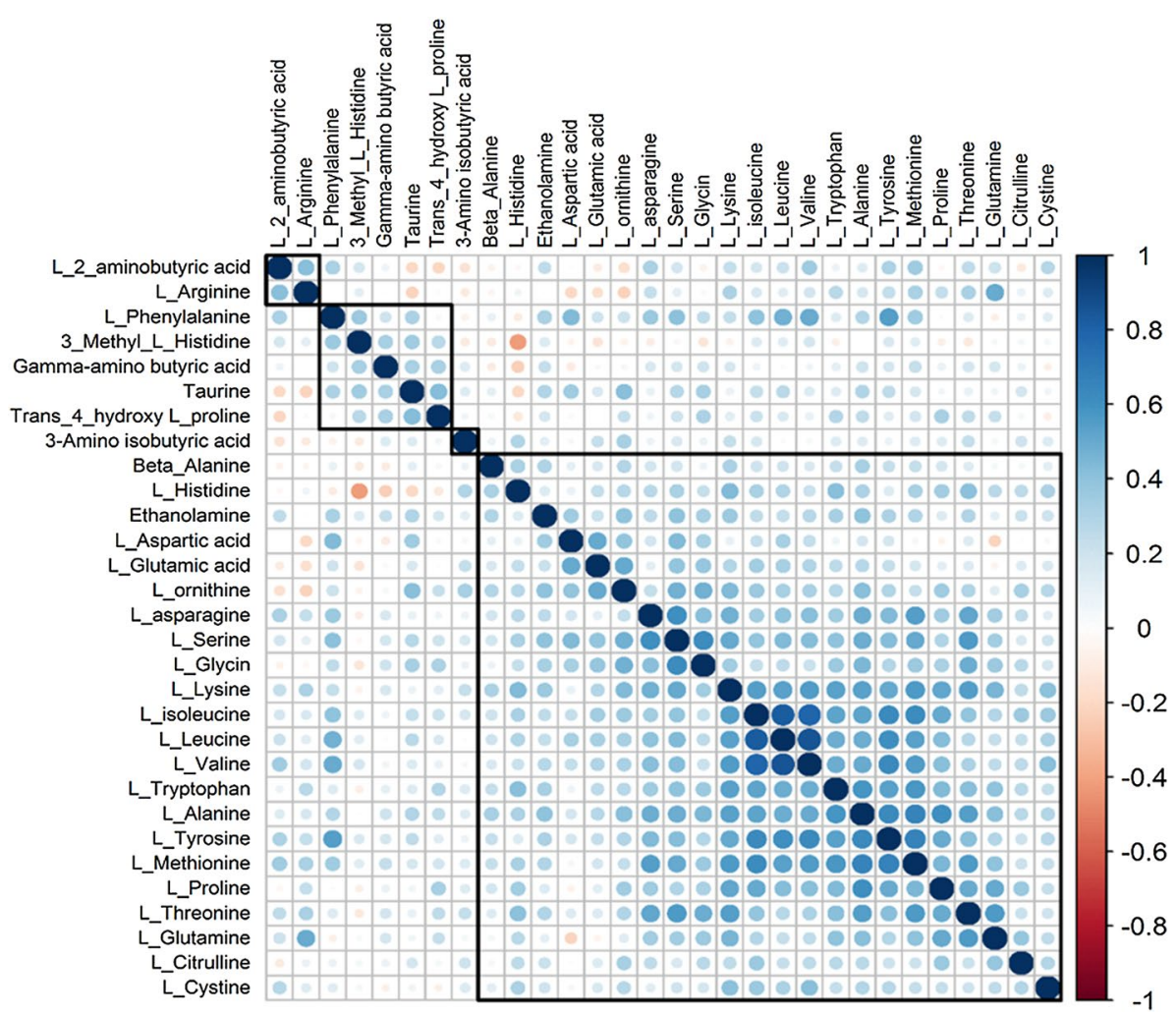


Fig. 5 Box plots graphs showing differences amino acid levels a Taurine, b Trans-4-hydroxy-L-proline and $\mathbf{c}$ L-Proline
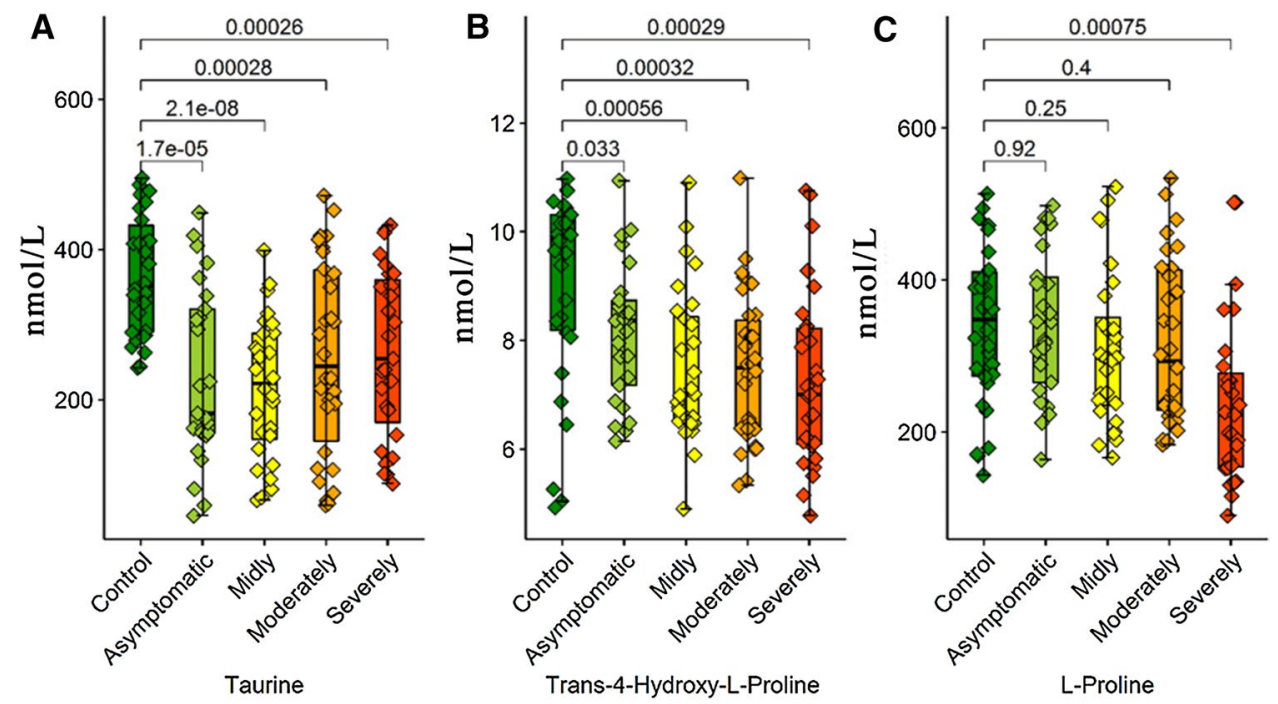

Fig. 6 Box plots graphs showing differences amino acid levels a L-Threonine and $\mathbf{b}$ L-Glutamine.
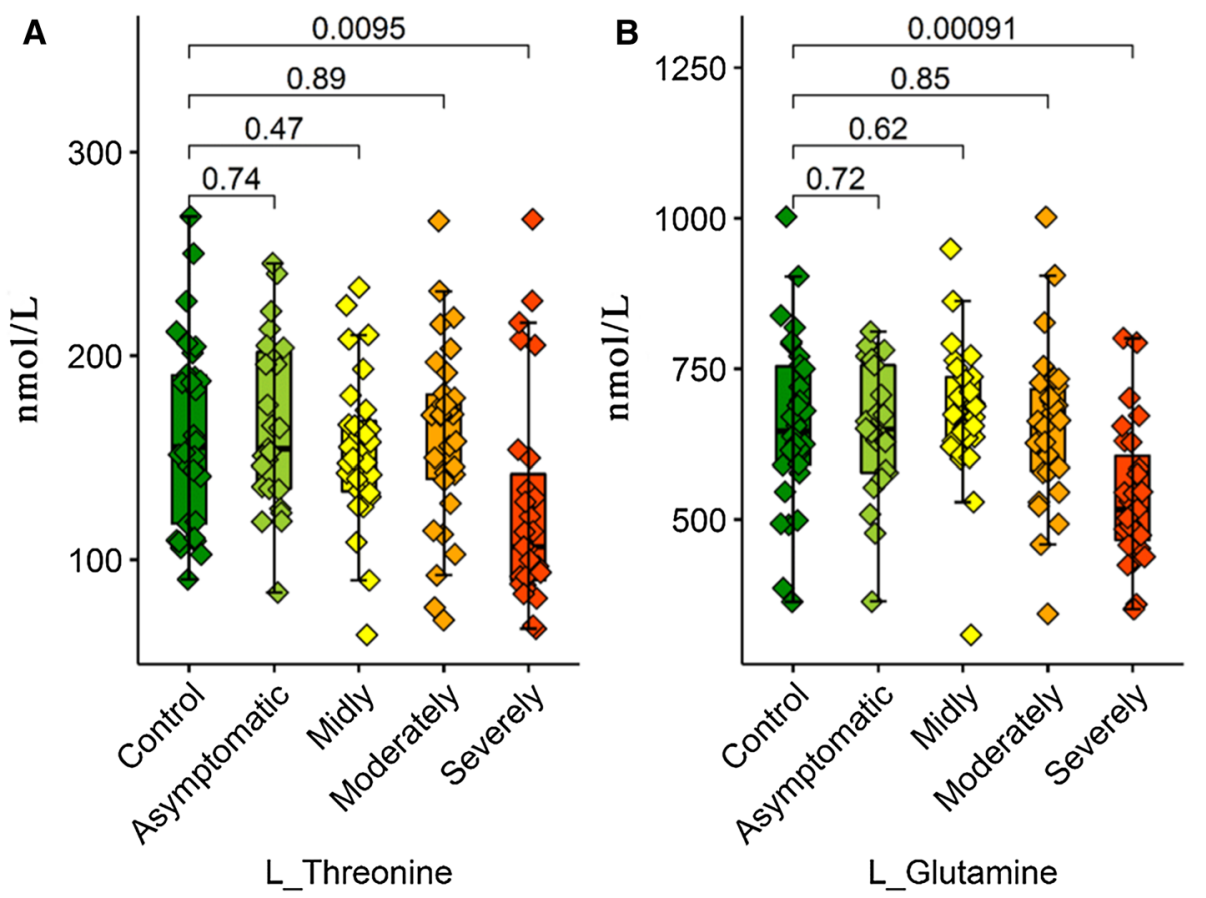

has also been observed in correlation with the severity of the disease (Fig. 8). This situation puts forward L-phenylalanine and L-2-aminobutyric acid as a potential target for differential diagnosis. In conclusion future clinical studies determining L-phenylalanine and L-2-aminobutyric levels can be used in laboratories as a COVID-19-biomarker. Also, supplementing COVID patients with taurine and BCAAs can be beneficial for treatment protocols. However, clinical and experimental studies with wider scope are needed to support these findings. 
Fig. 7 Box plots graphs showing differences amino acid levels a L-Histidine and $\mathbf{b}$ L-Citrulline

Fig. 8 Box plots graphs showing differences amino acid levels a L-Phenylalanine and $\mathbf{b}$ L-2-aminobutyric acid

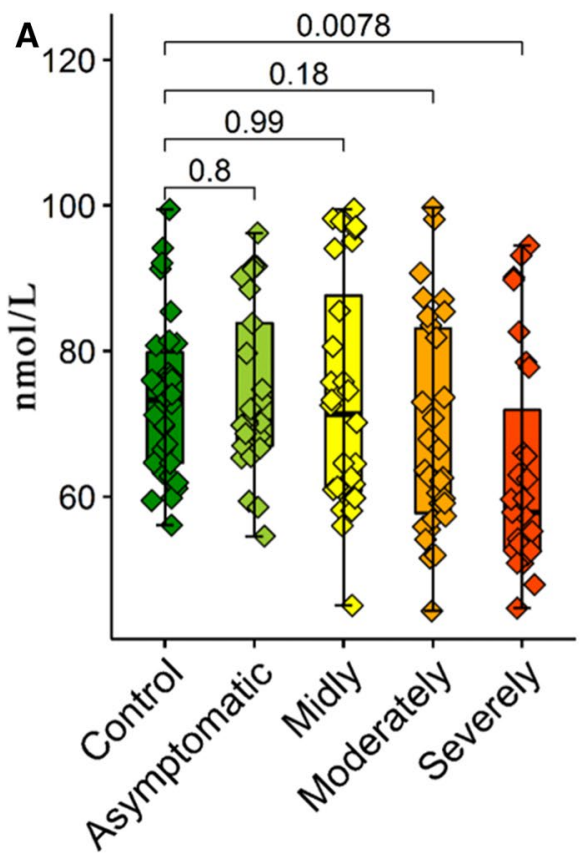

L_Histidine

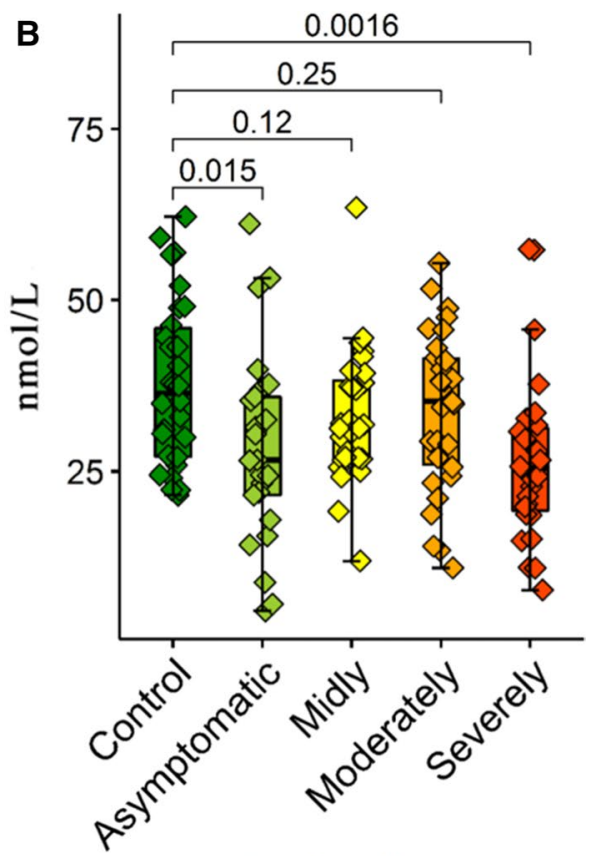

L_Citrulline
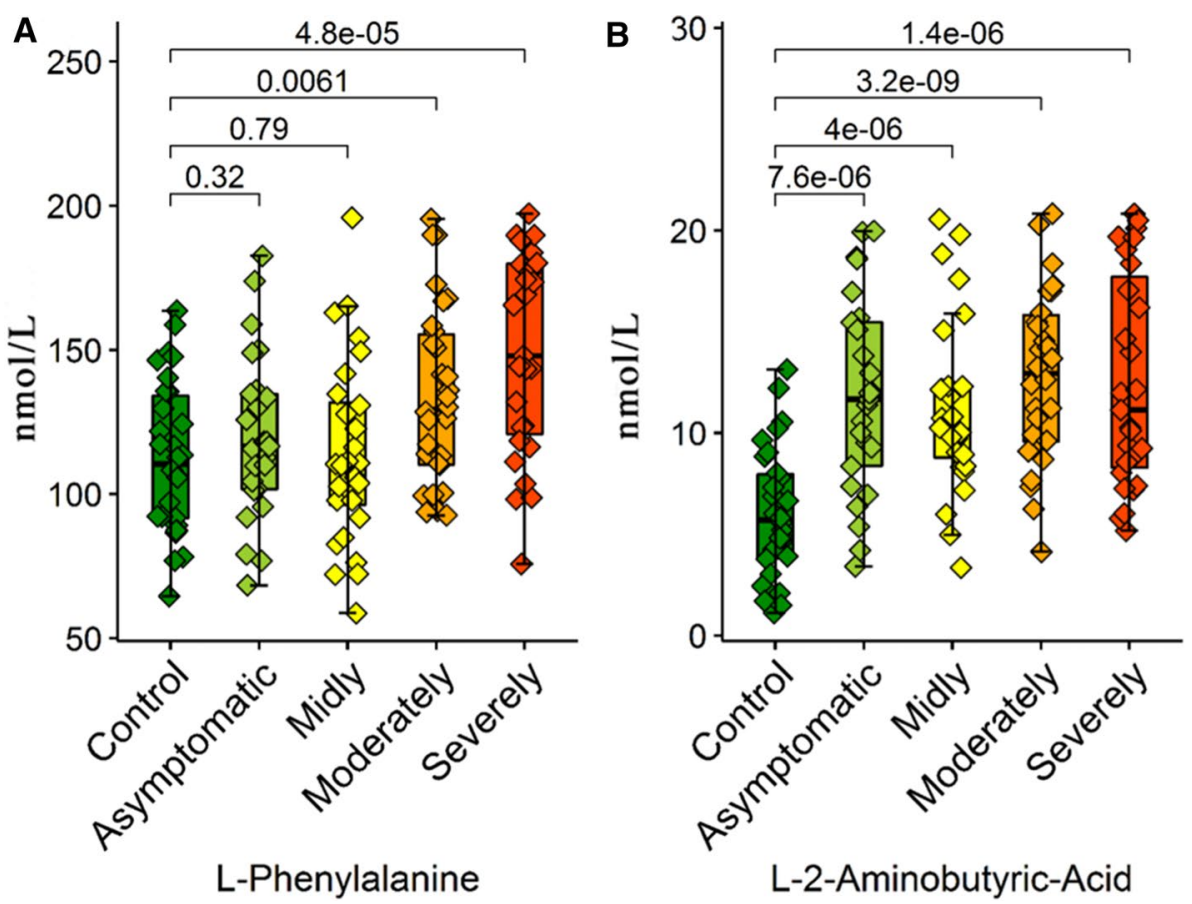
Supplementary Information The online version contains supplementary material available at https://doi.org/10.1007/s00726-021-03081-w.

\section{Declarations}

Conflicts of interest The authors declare no conflicts of interest.

Ethical standard The study was approved with Decision No.B.30.2.ATA.0.01.00/354 of the Ataturk University Medicine Faculty Clinical Research Ethical Board Commission.

\section{References}

Ardawi MSM, Newsholme EA (1983) Glutamine metabolism in lymphocytes of the rat. Biochem J 212(3):835-842

Bansal V, Ochoa JB (2003) Arginine availability, arginase, and the immune response. Curr Opin Clin Nutr Metab Care 6(2):223-228

Bi X, Henry CJ (2017) Plasma-free amino acid profiles are predictors of cancer and diabetes development. Nutr Diabetes 7(3):e-249-e

Cascino A, Muscaritoli M, Cangiano C, Conversano L, Laviano A, Ariemma $S$ et al (1995) Plasma amino acid imbalance in patients with lung and breast cancer. Anticancer Res 15(2):507-510

Celik M, Şen A, Koyuncu İ, Gönel A (2019) Plasma-free amino acid profiling of nasal polyposis patients. Comb Chem High Throughput Screen 22(9):657-662

Cengiz M, BorkuUysal B, Ikitimur H, Ozcan E, Islamoglu MS, Aktepe E et al (2020) Effect of oral 1-Glutamine supplementation on Covid-19 treatment. Clin Nutr Exp 33:24-31

Chen N, Zhou M, Dong X, Qu J, Gong F, Han Y et al (2020) Epidemiological and clinical characteristics of 99 cases of 2019 novel coronavirus pneumonia in Wuhan, China: a descriptive study. The Lancet 395(10223):507-513

Chiarla C, Giovannini I, Siegel JH (2011) Characterization of alpha-amino-n-butyric acid correlations in sepsis. Transl Res 158(6):328-333

Cui Y, Liu S, Zhang X, Ding X, Duan X, Zhu Z et al (2020) Metabolomic analysis of the effects of adipose-derived mesenchymal stem cell treatment on rats with sepsis-induced acute lung injury. Front Pharmacol 11:902

Dall'Igna DM, da Luz JM, Vuolo F, Klamt F, Dal Pizzol F (2013) Taurine chloramine decreases cell viability and cytokine production in blood and spleen lymphocytes from septic rats submitted to sepsis. Crit Care 17(4):1-59

Demopoulos C, Antonopoulou S, Theoharides TC (2020) COVID-19, microthromboses, inflammation, and platelet activating factor. BioFactors 46(6):927-933

Du X, Li Y, Wang Y, You H, Hui P, Zheng Y et al (2018) Increased branched-chain amino acid levels are associated with long-term adverse cardiovascular events in patients with STEMI and acute heart failure. Life Sci 209:167-172

Duval D, Demangel C, Munier-Jolain K, Miossec S, Geahel I (1991) Factors controlling cell proliferation and antibody production in mouse hybridoma cells: i. Influence of the amino acid supply. Biotechnol Bioeng 38(6):561-570

Effros RM (2011) Alpha aminobutyric acid, an alternative measure of hepatic injury in sepsis? Transl Res 158(6):326-327

Franěk F, Srámková K (1996) Protection of B lymphocyte hybridoma against starvation-induced apoptosis: survival-signal role of some amino acids. Immunol Lett 52(2-3):139-144

Hakuno D, Hamba Y, Toya T, Adachi T (2015) Plasma amino acid profiling identifies specific amino acid associations with cardiovascular function in patients with systolic heart failure. PLoS ONE 10(2):e0117325

Hasim A, Aili A, Maimaiti A, Abudula A, Upur H (2013) Plasma-free amino acid profiling of cervical cancer and cervical intraepithelial neoplasia patients and its application for early detection. Mol Biol Rep 40(10):5853-5859

Hiraiwa H, Okumura T, Kondo T, Kato T, Kazama S, Ishihara T et al (2020) Usefulness of the plasma branched-chain amino acid/ aromatic amino acid ratio for predicting future cardiac events in patients with heart failure. J Cardiol 75(6):689-696

Ho B, Ray A (2020) A case for palliative dermatology: COVID-19related dermatoses. Clin Dermatol 38(6):768-769

Holecek M (2010) Three targets of branched-chain amino acid supplementation in the treatment of liver disease. Nutrition 26(5):482-490

Holeček M (2018) Branched-chain amino acids in health and disease: metabolism, alterations in blood plasma, and as supplements. Nutr Metab 15(1):1-12

Hong S-Y, Yang D-H, Chang S-K (1998) The relationship between plasma homocysteine and amino acid concentrations in patients with end-stage renal disease. J Ren Nutr 8(1):34-39

Hoskin S, Bremner D, Holtrop G, Lobley G (2016) Responses in whole-body amino acid kinetics to an acute, sub-clinical endotoxin challenge in lambs. Br J Nutr 115(4):576-584

Huang S-S, Lin J-Y, Chen W-S, Liu M-H, Cheng C-W, Cheng M-L et al (2019) Phenylalanine-and leucine-defined metabolic types identify high mortality risk in patients with severe infection. Int J Infect Dis 85:143-149

Huang C, Wang Y, Li X, Ren L, Zhao J, Hu Y et al (2020) Clinical features of patients infected with 2019 novel coronavirus in Wuhan, China. The Lancet 395(10223):497-506

Innocenti GP, Santinelli L, Laghi L, Borrazzo C, Pinacchio C, Fratino $\mathrm{M}$ et al (2020) Modulation of phenylalanine and tyrosine metabolism in HIV-1 infected patients with neurocognitive impairment: results from a clinical trial. Metabolites 10(7):274

Kassambara A (2018) ggpubr:"ggplot2" based publication ready plots (Version 0.1. 7). https://CRAN.R-project.org/package=ggpubr

Kudsk KA (2006) Immunonutrition in surgery and critical care. Annu Rev Nutr 26:463-479

Leichtle AB, Nuoffer J-M, Ceglarek U, Kase J, Conrad T, Witzigmann $\mathrm{H}$ et al (2012) Serum amino acid profiles and their alterations in colorectal cancer. Metabolomics 8(4):643-653

Li H, Liu L, Zhang D, Xu J, Dai H, Tang N et al (2020) SARS-CoV-2 and viral sepsis: observations and hypotheses. The Lancet 395(10235): 1517-1520

Liu Y, Wang X, Hu C-AA (2017) Therapeutic potential of amino acids in inflammatory bowel disease. Nutrients 9(9):920

Liu J, Li S, Liu J, Liang B, Wang X, Wang H et al (2020) Longitudinal characteristics of lymphocyte responses and cytokine profiles in the peripheral blood of SARS-CoV-2 infected patients. EBioMedicine 55:102763

Manig F, Kuhne K, von Neubeck C, Schwarzenbolz U, Yu Z, Kessler $\mathrm{BM}$ et al (2017) The why and how of amino acid analytics in cancer diagnostics and therapy. J Biotechnol 242:30-54

Mayer P (2011) Multivariate Grafiken für PLS Pfadmodelle: Institut für Statistik

Mayers JR, Wu C, Clish CB, Kraft P, Torrence ME, Fiske BP et al (2014) Elevation of circulating branched-chain amino acids is an early event in human pancreatic adenocarcinoma development. Nat Med 20(10):1193-1198

Mierzchala-Pasierb M, Lipinska-Gediga M, Fleszar MG, Lesnik P, Placzkowska S, Serek P et al (2020) Altered profiles of serum amino acids in patients with sepsis and septic shock-preliminary findings. Arch Biochem Biophys 691:108508 
Mondanelli G, Iacono A, Carvalho A, Orabona C, Volpi C, Pallotta MT et al (2019) Amino acid metabolism as drug target in autoimmune diseases. Autoimmun Rev 18(4):334-348

Mustafa A, Gupta S, Hudes GR, Egleston BL, Uzzo RG, Kruger WD (2011) Serum amino acid levels as a biomarker for renal cell carcinoma. J Urol 186(4):1206-1212

Nair MS, Yao D, Chen C, Pieters M (2019) Serum metabolite markers of early Mycoplasma hyopneumoniae infection in pigs. Vet Res 50(1):1-10

Newsholme P, Newsholme EA (1989) Rates of utilization of glucose, glutamine and oleate and formation of end-products by mouse peritoneal macrophages in culture. Biochem J 261(1):211-218

Newsholme P, Curi R, Curi TP, Murphy C, Garcia C, De Melo MP (1999) Glutamine metabolism by lymphocytes, macrophages, and neutrophils: its importance in health and disease. J Nutr Biochem 10(6):316-324

Organization WH (2021) WHO Coronavirus Disease (COVID-19) Dashboard 2021, February 2. https://covid19. who.int/table

Paez-Franco JC, Torres-Ruiz J, Sosa-Hernandez VA, Cervantes-Diaz R, Romero-Ramirez S, Perez-Fragoso A et al (2021) Metabolomics analysis reveals a modified amino acid metabolism that correlates with altered oxygen homeostasis in COVID-19 patients. Sci Rep 11(1):6350

Phang JM (1985) The regulatory functions of proline and pyrroline5-carboxylic acid. CurrTop Cell Regul. 25:91-132

Philips AM, Khan N (2021) Amino acid sensing pathway: a major check point in the pathogenesis of obesity and COVID-19. Obes Rev 22(4):e13221

Picca A, Calvani R, Landi G, Marini F, Biancolillo A, Gervasoni J et al (2019) Circulating amino acid signature in older people with Parkinson's disease: a metabolic complement to the EXosomes in PArkiNson Disease (EXPAND) study. Exp Gerontol 128:110766

Repubic of Turkey, Ministry of Health (2020) SARS-CoV-2 Infection, Adult patients treatment guideline. https://covid19.saglik.gov.tr/ Eklenti/40719/0/covid-19rehberieriskinhastayonetimivetedavipdf. pdf

Reeds P, Jahoor F (2001) The amino acid requirements of disease. Clin Nutr 20:15-22

Rees CA, Rostad CA, Mantus G, Anderson EJ, Chahroudi A, Jaggi P et al (2021) Altered amino acid profile in patients with SARSCoV-2 infection. Proc Natl Acad Sci USA 118(25):1-3

Richman DD, Whitley RJ, Hayden FG (2020) Clinical virology. Wiley

Sakurai A, Sasaki T, Kato S, Hayashi M, Tsuzuki S-I, Ishihara T et al (2020) Natural history of asymptomatic SARS-CoV-2 infection. N Engl J Med 383(9):885-886

Shi Y, Wang G, Cai X-p, Deng J-W, Zheng L, Zhu H-H et al (2020) An overview of COVID-19. J Zhejiang Univ Sci B 21(5):343-360

Sookoian S, Pirola CJ (2014) Metabolic make-up of NASH: from fat and sugar to amino acids. Nat Rev Gastroenterol Hepatol 11(4):205-207

Spittler A, Oehler R, Goetzinger P, Holzer S, Reissner CM, Leutmezer $F$ et al (1997) Low glutamine concentrations induce phenotypical and functional differentiation of U937 myelomonocytic cells. J Nutr 127(11):2151-2157

Su J, Zhu Y, Jiang Y, Zou L, Liu X, Xu Y (2017) Study of Plasma amino acid related metabolites of septic rats using gas chromatography-mass spectrometry. Zhonghua Wei Zhong Bing Ji Jiu Yi Xue 29(4):332-336
Tochikubo O, Nakamura H, Jinzu H, Nagao K, Yoshida H, Kageyama $\mathrm{N}$ et al (2016) Weight loss is associated with plasma free amino acid alterations in subjects with metabolic syndrome. Nutr Diabetes 6(2):e-197-e

Uzzan M, Soudan D, Peoc'h K, Weiss E, Corcos O, Treton X (2020) Patients with COVID-19 present with low plasma citrulline concentrations that associate with systemic inflammation and gastrointestinal symptoms. Dig Liver Dis 52(10):1104-1105

Vente JP, von Meyenfeldt MF, Van Eijk H, Van Berlo C, Gouma DJ, van der Linden CJ et al (1989) Plasma-amino acid profiles in sepsis and stress. Ann Surg 209(1):57

Vliegen G, Kehoe K, Bracke A, De Hert E, Verkerk R, Fransen E et al (2020) Dysregulated activities of proline-specific enzymes in septic shock patients (sepsis-2). PLoS ONE 15(4):e0231555

Wang TJ, Larson MG, Vasan RS, Cheng S, Rhee EP, McCabe E et al (2011) Metabolite profiles and the risk of developing diabetes. Nat Med 17(4):448-453

Watanabe A, Higashi T, Sakata T, Nagashima H (1984) Serum amino acid levels in patients with hepatocellular carcinoma. Cancer 54(9): 1875-1882

Wu G (1997) Synthesis of citrulline and arginine from proline in enterocytes of postnatal pigs. Am J Physiol-Gastrointest Liver Physiol 272(6):G1382-G90

Wu G (2009) Amino acids: metabolism, functions, and nutrition. Amino Acids 37(1):1-17

Wu Z, McGoogan JM (2020) Characteristics of and important lessons from the coronavirus disease 2019 (COVID-19) outbreak in China: summary of a report of 72314 cases from the Chinese Center for Disease Control and Prevention. JAMA 323(13):1239-1242

Wu G, Morris SM Jr (1998) Arginine metabolism: nitric oxide and beyond. Biochem J 336(1):1-17

Wu G, Field C, Marliss EB (1991) Elevated glutamine metabolism in splenocytes from spontaneously diabetic BB rats. Biochem $\mathrm{J}$ 274(1):49-54

Wu G, Field CJ, Marliss EB (1992) Enhanced glutamine and glucose metabolism in cultured rat splenocytes stimulated by phorbol myristate acetate plus ionomycin. Metabolism 41(9):982-988

Wu G, Bazer FW, Hu J, Johnson GA, Spencer TE (2005) Polyamine synthesis from proline in the developing porcine placenta. Biol Reprod 72(4):842-850

Yanagisawa R, Kataoka M, Inami T, Momose Y, Kawakami T, Takei $M$ et al (2015) Usefulness of circulating amino acid profile and Fischer ratio to predict severity of pulmonary hypertension. Am J Cardiol 115(6):831-836

Yaqoob P, Calder PC (1998) Cytokine production by human peripheral blood mononuclear cells: differential senstivity to glutamine availability. Cytokine 10(10):790-794

Yufang S, Ying W, Changsun S (2020) COVID-19 infection: the perspective on immune response. Cell Death Differ 27(5):1451-1454

Zhou F, Yu T, Du R, Fan G, Liu Y, Liu Z et al (2020) Clinical course and risk factors for mortality of adult inpatients with COVID19 in Wuhan, China: a retrospective cohort study. The Lancet 395(10229):1054-1062

Publisher's Note Springer Nature remains neutral with regard to jurisdictional claims in published maps and institutional affiliations. 


\section{Authors and Affiliations}

\section{Alptug Atila ${ }^{1}$ (1) $\cdot$ Handan Alay ${ }^{2} \cdot$ Mehmet Emrah Yaman $^{1} \cdot$ Tugrul Cagri Akman $^{3} \cdot$ Elif Cadirci $^{4} \cdot$ Burak Bayrak $^{1}$. Saffet Celik ${ }^{5} \cdot$ Nihal Efe Atila ${ }^{6} \cdot$ Aycan Mutlu Yaganoglu ${ }^{7} \cdot$ Yucel Kadioglu $^{1} \cdot$ Zekai HalıcI $^{4} \cdot$ Emine Parlak $^{2}$. Zafer Bayraktutan ${ }^{8}$}

Handan Alay

handan.alay@atauni.edu.tr

Mehmet Emrah Yaman

emrahyaman@atauni.edu.tr

Tugrul Cagri Akman

tugrul.akman@erzincan.edu.tr

Elif Cadirci

ecadirci@atauni.edu.tr

Burak Bayrak

burak.bayrak@atauni.edu.tr

Saffet Celik

saffetcelik@trakya.edu.tr

Nihal Efe Atila

nihalefe24@hotmail.com

Aycan Mutlu Yaganoglu

myagan@atauni.edu.tr

Yucel Kadioglu

yucel@atauni.edu.tr

Zekai Halıcı

hzekai@gmail.com

Emine Parlak

emine.parlak@atauni.edu.tr
Zafer Bayraktutan

zafer.bayraktutan@atauni.edu.tr

1 Department of Analytical Chemistry, Faculty of Pharmacy, Ataturk University, 25240 Erzurum, Turkey

2 Department of Infectious Diseases and Clinical Microbiology, Ataturk University Faculty of Medicine, 25240 Erzurum, Turkey

3 Department of Analytical Chemistry, Faculty of Pharmacy, Erzincan Binali Yildirim University, 25240 Erzurum, Turkey

4 Department of Pharmacology, Ataturk University Faculty of Medicine, 25240 Erzurum, Turkey

5 Technology Research and Development Application and Research Center, Trakya University, 22030 Edirne, Turkey

6 Department of Otorhinolaryngology, Erzurum Regional Training and Research Hospital, 25240 Erzurum, Turkey

7 Department of Animal Science, College of Agriculture, Ataturk University, 25240 Erzurum, Turkey

8 Department of Biochemistry, Ataturk University Faculty of Medicine, 25240 Erzurum, Turkey 